\section{MUERTE NO ACCIDENTAL DE UN ANARQUISTA ESPAÑOL: EL PERIODISTA Y ESCRITOR BENIGNO BEJARANO MUERE EN UN CAMPO DE EXTERMINIO}

\author{
Julia María Labrador Ben \\ Universidad Complutense de Madrid \\ julia9254@yahoo.es
}

\section{NO ACCIDENTAL DEATH OF A SPANISH ANARCHIST: THE JOURNALIST AND WRITER BENIGNO BEJARANO DIED IN AN EXTERMINATION CAMP}

\begin{abstract}
The journalist and anarchist writer, Benigno Bejarano (Alburquerque, Badajoz, 22.XI.1900 - ¿Watenstedt?, Germany, summer 1944) developed an intensive political and literary career before the Spanish Civil War and also during that War. He went to France into exile and was arrested by the Gestapo and then sent to different concentration camps. Information is given on his biography, his works, his exile and also his arrest and his detention until he was gassed by the Nazis in a ghost truck. An exhaustive bibliography is also added to complete this study.
\end{abstract}

KEY WORDS: Benigno Bejarano; Lazarillo de Tormes (pseudonym); spanish anarchism; republican press and anarcho-syndicalism; Lecturas; Solidaridad Obrera; España Nueva; humour; political-literary satire; extermination camps.

\section{BiografíA}

Sobre la vida de Benigno Bejarano Domínguez apenas se conocen datos. Nació en Alburquerque (Badajoz) el 22 de noviembre de 1900, hijo de Segundo Bejarano y Petra Domínguez. Fue el menor de tres hermanos'. Por su hoja de reclutamiento sabemos que era moreno, con el pelo y los ojos negros, medía escasamente 1,72 metros y su perímetro torácico era reducido, 75 centímetros, una complexión física extremadamente débil que le impidió soportar los trabajos forzados a los que fue obligado al final de su vida. Esta descripción física coincide con la que da Abel Paz en el segundo tomo de sus memorias, en las que recuerda también su carácter irónico y su interés por los juegos con el lenguaje, rasgos que estarán muy presentes en toda su narrativa:

Bejarano era un tipo delgado, sumamente delgado, alto, de cara alargada, como escapada de un cuadro de El Greco. Cabello negro ensortijado y una frente abombada, extremadamente abombada. En su mirada titileaba siempre una brizna de ironía que hacía difícil saber cuándo realmente
RESUMEN: El periodista y escritor anarquista Benigno Bejarano (Alburquerque, Badajoz, 22-XI-1900 - ¿Watenstedt?, Alemania, verano 1944) desarrolló una intensa labor literaria y política antes y durante la Guerra Civil española. Exiliado en Francia, es detenido por la Gestapo y trasladado a varios campos de concentración. Se da noticia de su biografía, sus obras, su exilio y su detención y reclusión hasta su muerte, gaseado en un camión fantasma por los nazis. Completa el estudio una exhaustiva bibliografia.

PALABRAS CLAVE: Benigno Bejarano; Lazarillo de Tormes (seudónimo); anarquismo español; prensa republicana y anarcosindicalista; Lecturas; Solidaridad Obrera; España Nueva; humorismo; sátira político-literaria; campos de exterminio.

bromeaba o hablaba en serio. Gustaba de los juegos de palabras en un castellano elegante y muy bien hablado, pese a su origen extremeño (Paz, 1993, 163).

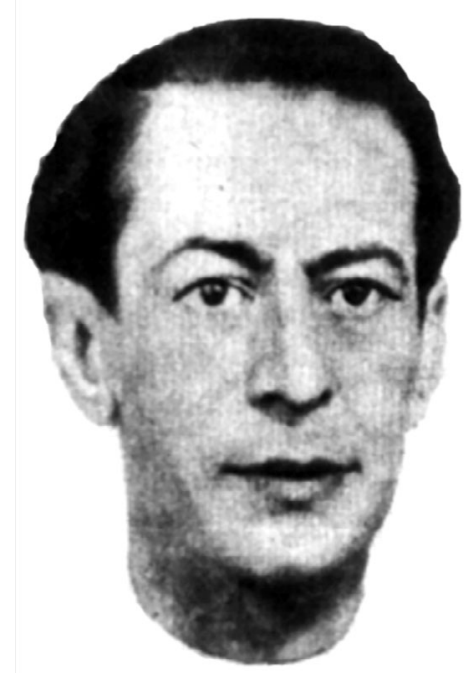

Fotografía de Benigno Bejarano publicada en prensa en 1938. 
Aunque en el año de su alistamiento y en la revisión de 1922 fue excluido del servicio militar, ingresó en caja como soldado el 1 de agosto de $1923^{2}$ en Alburquerque, y el 8 de febrero de 1924 fue destinado al Regimiento de Infantería de Vergara número 57 formando parte del cupo de filas. Durante su etapa como recluta tuvo problemas con el ejército: se le indultó en septiembre de 1924, siendo cabo se fugó del Regimiento de Infantería de Vergara y fue detenido en agosto de 1925, e indultado nuevamente en julio de 1927. Periodista ${ }^{3}$, escritor y militante anarquista, fue estudiante en Paris y participó activamente durante la Guerra Civil española.

Exiliado en Francia desde 1939, en 1941 conocerá en Burdeos a Abel Paz, quien recuerda, de nuevo en sus memorias, que Bejarano vivía con su mujer ${ }^{4}$ en un hotel cercano al bar o "bistrot" Chez Pierre de la pequeña calle Moliner, en el que se hospedaban también Antonio Casanova y el pintor y escultor Eleuterio Blasco Ferrer ${ }^{5}$. La Gestapo lo detiene en 1942 y lo encarcela en el castillo de HA de Bordieux. En 1943 será

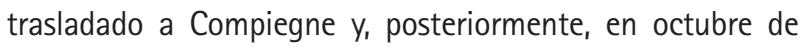
ese mismo año, confinado en el campo de concentración de Neuengamme, del que pasará a Watenstedt, un campo comando del anterior en el que fue asignado al grupo constructor de barracones de ladrillo y cemento. Empedernido fumador, su salud estaba muy quebrantada por la rudeza de tales trabajos, tanto que empezó a escupir sangre; durante un tiempo Enrique Comas, con la ayuda de un doctor polaco, lo retuvo en la enfermería donde se encargó de pintar puertas e interiores, hasta que los nazis se dieron cuenta de su precario estado y decidieron llevárselo a "trabajos menos pesados", eufemismo que encubría ser destinado a la cámara de gas y los hornos crematorios. Murió gaseado por los nazis en un camión fantasma en el verano de $1944^{6}$.

En 1932 Alfonso Martínez Rizo publicó una novela titulada 1945. El advenimiento del comunismo libertario ${ }^{7}$, ficción futurista en la que Bejarano aparece como personaje y que, a la luz de los desgraciados acontecimientos que acabamos de exponer, se vuelve imposible porque en ese año de 1945 ya había fallecido.

\section{SU OBRA LITERARIA}

Si de su vida, como ya hemos dicho, apenas conocemos datos, tampoco sabemos cómo irrumpió exactamente en el mundo literario, debido, entre otras cosas, a una escasez de testimonios, ya que la información de prensa de su momento se limitaba a reseñar sus libros y su inclusión en los estudios literarios posteriores sobre esa época es reducidísima. Cabe dividir su obra en tres etapas: la anterior a la proclamación de la Segunda República, es decir, a 1931; sus escritos durante el período republicano, 1931 a 1936; y por último, los aparecidos durante la Guerra Civil.

Su primera actividad fue de tipo periodístico ${ }^{8}$ en diarios como El Progreso o España Nueva, y en revistas como Lecturas y Estudios. Revista ecléctica. Curiosamente, en los artículos de esta última cabe presuponer una cierta formación científica en Bejarano, que le permite tratar temas de biología y medicina en consonancia con la temática general abordada por la revista. En Lecturas, aparte de algunas contribuciones breves de carácter literario, en concreto siete cuentos cómicos aparecidos entre 1929 y 1932, publicó un texto más importante, El secreto de un loco, una de sus primeras novelas, como inserto encuadernable a manera de folletín de la revista (Labrador Ben, 2009). Trinidad Labajo se refiere a Benigno Bejarano con la denominación "el gran imaginativo" en el capítulo dedicado a colaboradores dentro de su estudio sobre Lecturas (Labajo González, 2003, 66), calificación que se puede completar con otra, el gran sarcástico.

Entre sus primeros libros figuran La mujer enigma, La huella heráldica, Historia de don Silvio de Alburquerque, El hombre que vendía la camisa y El caso del Doctor González.

Aunque no es éste el momento de abordar su creación desde el punto de vista literario, sí hemos de señalar que Bejarano lleva hasta el máximo el absurdo, algo que estilísticamente es muy estimable, y que no se arredra ante ningún género, ni siquiera ante la ciencia ficción. Así, El secreto de un loco se desarrolla en el planeta Marte. Marcianos, fantasmas y revolucionarios se dan la mano en sus textos. Fulgencio Castañar, autor de dos artículos muy interesantes sobre Bejarano, describe su primera época de la siguiente forma:

En todas las obras de este momento predomina el objetivo de provocar la risa. Los temas son enfocados desde una perspectiva grotesca; los personajes son envueltos en situaciones de ingenioso enredo, cuyo desenlace es imprevisible para el lector por la continua distorsión que hace Bejarano 
de las posibles soluciones racionales. La trama es presentada a través de un caleidoscopio que, por las inesperadas concatenaciones de causas, impide prever el final de la narración (Castañar, 1996, 155).

Castañar define esa etapa como humor evasivo, algo que cambiará radicalmente tras la proclamación de la Segunda República, debido a que ya no va a estar supeditado a la censura monárquica del período de Primo de Rivera.

El primer artículo de Bejarano en la prensa anarco-sindicalista aparece en Solidaridad Obrera el 24 de julio de 1931. Eso no significa que no participara antes en órganos de prensa de izquierdas, en concreto sabemos que en 1919 la CNT decide contratar un espacio en las páginas del periódico republicano España Nueva de Madrid, dirigido por Rodrigo Soriano, y Bejarano será uno de los designados para colaborar en él. Al año siguiente, en febrero de 1932, el Comité Regional le nombra redactor de dicha revista. Seguidor de Ángel Pestaña, abandona Solidaridad Obrera en 1933 y se integra en Cultura Libertaria.

Su libro más importante de ese período es Turistas en España. Novela epigramática, fechada su finalización por el autor en octubre de $1932^{9}$; escasamente cuatro años más tarde, en enero de 1936, Bejarano terminó su segunda parte titulada Los Caballeros del Bienio. Sátira. En la propaganda que sobre la primera parte se publicó en Solidaridad Obrera se definía a Bejarano como "el espíritu mordaz de Swift al servicio de la Revolución Social". Ese libro alcanzó un éxito notable, como el resto de sus obras, de las cuales se tiraron varias ediciones.

Efectivamente, durante todo ese período, tanto en sus libros como en sus artículos, Benigno Bejarano puso su pluma al servicio del anarco-sindicalismo. Esto le llevó a una lucha profunda contra el Estado. Bejarano plantea que, pese al cambio de régimen y la sustitución de la monarquía por la república, el Estado sigue teniendo los mismos componentes represivos y la misma estructura caciquil. Va a utilizar, como hará posteriormente en uno de sus libros más importantes, España cuna de la libertad. La revolución española y sus conflictos (1937), una crítica acerba a los sucesivos gobiernos de la República, aunque esa crítica se intensificará todavía más al juzgar el Bienio Negro y algunas figuras del mismo como Alejandro Lerroux.
De Turistas en España se hicieron seis ediciones, y algunas de las críticas sobre la misma pueden leerse al final de su libro Conspiradores, que incluía dos textos fechados en septiembre de 1930 en Paris, la novela que le da título y otra narración más corta, La herencia de mi tío. Dichas críticas fueron publicadas en El Diluvio, Heraldo de Madrid, EI Sol, La Tierra y Cultura Libertaria. Una vez más Castañar dice al respecto de Turistas en España:

La novela gozó de un éxito notable entre los sectores anarco-sindicalistas hasta el extremo de que el día 22 de julio de 1933, en un festival organizado por el Ateneo Sindicalista Libertario, en su sede Pueblo Nuevo, con el fin de obtener fondos para su supervivencia, Ángel Pestaña dio una conferencia sobre "Cómo debe ser nuestro teatro" y se ilustró con una versión escénica de Turistas... realizada por el propio Bejarano (Castañar, 1996, 161).

Con esto concluye su segunda etapa. La tercera estará dedicada a la lucha política durante el período de la Guerra Civil, Bejarano cambiará de nombre literario y adoptará el seudónimo de Lazarillo de Tormes. Va a alternar la pluma y la sátira política con la bomba y el fusil, convirtiéndose no sólo en un agitador literario, sino en un activista político. En el prólogo de España tumba del fascismo (Apuntes de un beligerante) (libro finalizado inicialmente en julio de 1937, pero no publicado hasta noviembre de ese mismo año), firmado por las Oficinas de Información, Propaganda y Prensa del Comité Nacional de la CNT, se dice lo siguiente: "'Lazarillo de Tormes' no ha escrito estas páginas con pluma elegante [...] lo ha hecho con la bomba y el fusil" (p. ???).

Y efectivamente, Bejarano toma parte en la contienda desde el primer momento, como relata en ese libro al hablar del 19 de julio de 1936 en Madrid y de los tiroteos en la Gran Vía y la toma del Cuartel de la Montaña.

España tumba del fascismo consta de veinte capítulos precedidos por el Prólogo antes citado y continuados en un epílogo y un apéndice. El libro, en cuya portada aparecen una fosa y una calavera con una esvástica dibujada en el hueso frontal que es lanzada por una pala sobre un montón de huesos, da comienzo con la "Defunción del hombre nietzschiano, Al socialismo por el hambre, Al fascismo por necesidad (naturalmente de la burguesía y como ideología de combate frente a la Revolución Proletaria), Panorama de Europa, y España". El siguiente capítulo trata de la Revolu- 
ción del 4 de octubre de 1934, en él se vierten durísimas críticas contra la represión ejercida por el gobierno republicano. Continúa después con el análisis de las elecciones de febrero de 1936 que dieron el triunfo al Frente Popular, los asesinatos -los denomina muy acertadamente desde un punto de vista etimológico- del teniente Castillo y de Joaquín Calvo Sotelo, la sublevación del 19 de julio en Madrid, en cuya represión toma parte como ya se ha dicho; y finaliza haciendo alusión a las intervenciones italiana y alemana, aseverando que el fascismo será derrotado en España y en Europa. El apéndice final está dirigido a un hipotético camarada que vive fuera de España, en Latinoamérica, al que describe la realidad española.

Con idénticos planteamientos ideológicos publicará, durante la Guerra Civil, dos libros más: Enviado especial. Novela, dividida en veinticinco capítulos y un epílogo, publicada en Barcelona en 1938, y otro libro mucho más importante, España cuna de la libertad. La revolución española y sus conflictos, publicado en Valencia en agosto de 1937. De nuevo el texto se estructura en quince capitulos y un epílogo. Toda la obra está dedicada a combatir al Partido Comunista de España y, por extensión, al movimiento comunista internacional. La afirmación más tajante de Bejarano es que la fuerza política preponderante en España, y la única revolucionaria, es el anarquismo.

El libro se estructura en siete apartados, cada uno con dos capítulos (salvo el tercero, con tres), según el siguiente esquema:

La revolución de las Ideas (capitulos 1-2).

La revolución en la Calle (capitulos 3-4).

La revolución en el Estado (capítulos 5-7).

La revolución en la Política (capitulos 8-9).

La revolución en la Intriga (capítulos 10-11).

La revolución en el Lodo (capitulos 12-13).

La revolución en Peligro (capitulos 14-15).

Una parte importante de la obra está dedicada a analizar el proceso y la represión del POUM (Partido Obrero de Unificación Marxista) y la desaparición y posterior liquidación de Andrés Nin. Aquí Bejarano utiliza el sarcasmo al máximo, en especial al analizar la calificación de "aliado del fascismo" que los comunistas aplicaban a León Trostky. Su conclusión no pude ser más contundente: "El Frente Popular y el Partido Comunista de España no son REVOLU-
CIONARIOS" (135). Siguiendo la pauta de su libro anterior, concluye con un epilogo en forma de carta dirigida a su amigo A. X. que milita en el Partido Comunista.

El tercer libro es una novela, Enviado especial, editada por el autor en Barcelona y fechada también en 1938. Consta de veinticinco capítulos y un epílogo. Esta obra es calificada por Bejarano como "sátira". Fulgencio Castañar, en su artículo reiteradamente citado, informa sobre el contenido:

\footnotetext{
Bejarano construye la que nosotros consideramos su última obra, Enviado especial (1938), mediante la yuxtaposición de episodios levemente entrelazados por el hilo conductor del protagonista, personaje en el que, pese a las disquisiciones pirandelianas con el autor, encarna su propio punto de vista. Sorprende, de entrada, que en una novela escrita en plena Guerra Civil los asuntos aparezcan como reportajes sobre la vida en diversas ciudades europeas -aparentemente una frivolidad-y que, pese a la tragedia cotidiana, emplee el humor como ingrediente principal.

Mas al avanzar en la lectura se advierte que con el tratamiento de los asuntos desde una perspectiva insólita, lo que pretende Bejarano es revelar al lector tanto el entramado de los regímenes fascistas como las entretelas de algunas organizaciones internacionales que influian, con su actuación, en el desarrollo de la guerra española. La pretensión del novelista no es otra que la de arrancar "la máscara carnavalesca" que encubre diariamente la realidad (pp. 161-162).
}

Bejarano lanza sus ataques a diestro y siniestro, y no le falta razón. La responsabilidad de que la República perdiera la guerra no fue únicamente de Mussolini y Hitler, sino también de la Sociedad de Naciones y del Comité de No Intervención.

Los planteamientos humanistas de Bejarano están bien definidos y se inscriben dentro del ideario anarquista. Optimista impenitente, no puede negar que la integración hombre-naturaleza postulada por Reclus estaba cada vez más lejos. Sólo el triunfo de la Revolución podría asegurarla.

\section{EXILIO Y MUERTE}

La guerra termina y Bejarano tiene que marchar al exilio, pero ni tan siquiera allí encontrará refugio. Su destino 
final, como el de muchos intelectuales españoles antifascistas, será la muerte a manos del nazismo.

Benito Bermejo, en su libro sobre el fotógrafo Francisco Boix (Bermejo, 2002, 50-59), analiza el proceso por el cual los españoles republicanos exiliados en Francia son internados en campos de concentración, algo que como ya dijimos al principio le sucedió al propio Bejarano. Internados inicialmente en campos de concentración franceses, al tener lugar la ocupación alemana son considerados, al principio, prisioneros de guerra y tratados conforme a dicho estatus. Estos primeros campos de detención a los que fueron trasladados, los Stalag, junto a los prisioneros de guerra franceses no resultaron demasiado duros. Pero muy pronto, los españoles fueron segregados por la Gestapo, que los identifican como Rotspanien, Rojos españoles, o mejor aún, Rotspanienkämpfer, combatientes de la España roja, a los que ya no cabía considerar como prisioneros de guerra. Luego serían enviados a diversos campos de concentración nazis, aunque en principio se les dijo que serían devueltos a España, lo cual quizá fue la primera intención de las autoridades alemanas. De hecho, algunos presos españoles muy significados como Lluis Companys, Presidente de la Generalitat catalana durante la guerra, fue apresado en Francia por la Gestapo y entregado a Franco que lo fusiló el 15 de octubre de 1940. Unos meses antes, a principios de agosto de ese mismo año, el primer grupo de trescientos noventa y dos presos españoles es internado en Mauthausen. Las familias fueron separadas y sólo los hombres, incluidos los mutilados y los niños mayores de trece años permanecieron internados en el campo. Las mujeres y los niños de menor edad fueron en muchos casos reenviados a España y entregados en Hendaya a las fuerzas policiales españolas. Hay que señalar que, desde el principio, estas actuaciones tuvieron la aquiescencia del Ministerio Español de Asuntos Exteriores. Los nazis se ofrecieron a prestar "toda la ayuda posible a la policía de seguridad española para la captura de los dirigentes rojos españoles" (Ministerio de Asuntos Exteriores, R. 1260, expediente 75). Del contingente de presos españoles murió un $87 \%$.

Pese a que algunos historiadores pongan en duda la actuación del Ministerio de Exteriores español en el proceso, lo cierto es que Serrano Suñer se entrevistó con Himmler y Heydrich y que el destino de los españoles fue establecido por una orden del propio Führer. Existe un documento muy ilustrativo que reproduce Bermejo, parte del discurso de August Eigruber, Gauleiter de Oberdonau, en cuya jurisdicción se encontraba Mauthausen:

Preguntad en Mauthausen, junto al Danubio: alli hay un gran campo de concentración. Hay 6.000 españoles rojos, esos revolucionarios españoles que se levantaron contra el fascista, Franco, y lucharon contra España por una España soviética. Ése era su eslogan. Eran dirigidos por generales de la Rusia soviética, oficiales de la Rusia soviética y lucharon con armas de la Rusia soviética. Y cuando España quedó derrotada y Franco venció, se exiliaron a Francia, y cuando ocupamos Francia el año pasado, el señor Pétain nos dio a esos 6.000 españoles rojos y declaró "No los necesito, no los quiero". Ofrecimos estos 6.000 españoles al jefe del estado Franco, el caudillo español. Rehusó y declaró que nunca admitiría a esos españoles rojos que lucharon por una España soviética. Entonces ofrecimos los 6.000 españoles rojos a Stalin y a la Rusia soviética, porque son luchadores por una revolución mundial, y el señor Stalin con su Komintern no los aceptó. Ahora están establecidos en Mauthausen estos 6.000 combatientes rojos, trabajadores -trabajadores de fábrica-; allí están para siempre. Al final no podemos iniciar una colonia española. No podemos asentarlos en ninguna parte. ¿Qué se supone que debemos hacer? (27 de junio de 1941) (Bermejo, 2002, 57).

El discurso es un prodigio de incongruencia y sus palabras son dichas apenas unos días después de la ruptura germano-soviética. Lo de los "rojos", genial invento del general Queipo de Llano, se aplicaba a tirios y troyanos, ya fueran socialistas, comunistas, anarquistas, o simplemente antifascistas. Por tanto, nada tiene de raro que los politicamente variopintos españoles fueran ofrecidos a Stalin quien, canallescamente, los rechazó. En el caso de Franco, tener que fusilar a seis mil españoles más no hacía más que incrementar un trabajo ya arduo. Y Pétain actuó como era de esperar, dejándolo todo en manos de sus aliados alemanes. Pero lo más impresionante de ese discurso es la afirmación de que "los revolucionarios españoles se levantaron contra el fascista, Franco, y lucharon contra España por una España soviética". Tampoco el jefe nazi tenía muy claro el concepto de España, pues dice: "Y cuando España quedó derrotada y Franco venció". Y realmente acierta: España fue derrotada y Franco, al que el término "fascista" no le hubiera gustado nada porque carecía de ideología, se instaló en el poder haciendo retroceder al país medio siglo. 
En alianza con la Francia colaboracionista y con los nazis decidió el exterminio de millares de españoles exiliados.

Para estos españoles recibí órdenes especiales; no podian escribir y nadie debía saber que se encontraban en el campo, dado que eran prisioneros de guerra franceses; habian tenido problemas con el gobierno de Vichy; para librase de ellos se había creado una comisión de liquidación en Berlín por orden de Serrano Suñer, Ministro de Relaciones Exteriores de España. La cesión data de 1941. Los españoles ya no debían existir. El comienzo había sido exitoso, pero estos españoles no eran tan fáciles de matar como los polacos (Bermejo, 2002, 58).

Los españoles no eran fáciles de matar, pero había que liquidarlos. Ése fue el caso de Benigno Bejarano. Los anarquistas españoles lo tuvieron muy difícil en el exilio. En Francia, la preponderancia del Partido Comunista Francés era notable y de éste salieron los miembros de la Resistencia, en la que se integraron muchos españoles que escaparon de los campos durante la desbandada del ejército francés ante el empuje alemán. En muchos casos, los jefes de los campos facilitaron la huida. Hay que señalar que la URSS se hizo cargo de muchos españoles, pero, naturalmente, sólo de aquellos que compartían la ideología comunista. Los anarquistas o los que no tuvieron la suerte de huir fueron llevados a campos de concentración, en el caso de Bejarano al de Neuengamme, situado junto a la orilla derecha del río Elba. Por alli pasaron cien mil prisioneros, el $80 \%$ de los cuales moriría de enfermedad, hambruna o simplemente asesinado. Tal fue el caso de nuestro autor, según relata, entre otros, Eduardo Pons Prades: "Benigno Bejarano, el popular novelista, que murió gaseado en un 'camión fantasma' en el invierno 1944-45" (Pons Prades, 2005, 105; y Pons Prades, 1995, 54).

La fecha es indeterminada ${ }^{10}$, pero por lo que sabemos, el avance aliado dio lugar a un repliegue inicial primero hacia el campo principal y luego a su total abandono ante la llegada de las tropas soviéticas por el este. Fue en esos momentos cuando tuvo lugar la matanza de prisioneros. A finales de abril, los aliados encontraron numerosas fosas llenas de cuerpos inánimes, que no pudieron, por falta de tiempo, ser cubiertas por los escasos supervivientes.
Benigno Bejarano murió, pues, gaseado por el tubo de escape del camión que ilusoriamente tendría que trasladarle a un destino más agradable. No hubiera corrido mejor suerte de haberse quedado en España o haber sido entregado a las autoridades franquistas. Habría sido internado en Porlier o en Montjüit, dependiendo de si hubiera sido apresado en Madrid o en Barcelona, y fusilado junto a las tapias del cementerio, como lo fueron algunos de sus camaradas de la acracia como Pedro Luis de Gálvez. Tampoco su calvario habría sido liviano e incluso hubiera tenido que asistir a misa. Pero lo que seguramente le habria indignado hasta la exasperación es que se le hubiera colgado el sambenito utilizado por los nazis al definir a los Rotspanienkämpfer: "españoles rojos que lucharon por una España soviética". Él jamás luchó por una sovietización del Estado español. Antes bien, su ideal revolucionario era muy distinto, el que correspondía al anarquismo. Claro que esas sutilezas no cabían en el pensamiento y en la literatura de combate de sus verdugos, ya fueran españoles, franceses colaboracionistas o alemanes.

\section{BIBLIOGRAFÍA DE Y SOBRE BENIGNO BEJARANO}

\section{Obras de Benigno Bejarano}

La mujer enigma, Novela.

La huella heráldica.

Historia de don Silvio de Alburquerque, Narración.

El hombre que vendía la camisa, Novela.

El caso del doctor González, Novela. Además se publicó como relato en la revista Lecturas, n. 131 (abril 1932), pp. 345-350.

(febrero-octubre 1930): El secreto de un loco: Novela, il. Serra Masana, Barcelona, Lecturas, Revista Literaria (Folletines de "Lecturas").

(1932): Fantasmas: relato, Barcelona, Ágora. La segunda edición fue comercializada por Ediciones Juvenal.

(1932): Turistas en España: Novela epigramática, Barcelona, Edición del autor, Gráficas Simó.

(1932): El fin de una expedición sideral (Viaje a Marte), BarceIona, Jiménez Letang (Colección Popular de Aventuras). Se comercializó con dos portadas diferentes: en la que apareció primero, el título se redujo sólo a Viaje a Marte, la ilustración bicolor era de Clavé y el precio 2 pesetas; en la segunda se amplió levemente el título a El fin de una expedición sideral. Viaje al planeta Marte, el ilustrador de la cuatricomía fue Tomàs y se aumentó su precio a 2,50 pesetas. 
(1933): Conspiradores, Novela, Barcelona, Juvenal. Incluye dos obras: Conspiradores y La herencia de mi tío.

(1936): Los Caballeros del Bienio: sátira, Málaga, Nueva Generación - Pedro Castro Domínguez. Según se indica en la portada externa, se trata de la 2. ${ }^{\text {a }}$ parte de Turistas en España.

Lazarillo de Tormes (Seud.) (1937): España cuna de la libertad: La revolución española y sus conflictos, Valencia, Ebro.

Lazarillo de Tormes (Seud.) (1937): España, tumba del fascismo, La guerra (Apuntes de un beligerante), Barcelona, Tierra y Libertad.

(1938): España frente al fascismo internacional: Acta procesal de la intervención extranjera en España, Barcelona, Ediciones del Comité Regional de la CNT en Cataluña, Sección de Propaganda y Prensa.

La epopeya española. Trilogía. Suponemos que estaba formada por los tres libros anteriores: España cuna de la libertad, España, tumba del fascismo y España frente al fascismo internacional.

(1938): El secreto de un loco, Novela, Barcelona, Maratón, Solidaridad Obrera. Se indica que es la cuarta edición.

(1938): Enviado especial, Novela, Barcelona, Edición del autor, Solidaridad Obrera.

\section{Obras de Benigno Bejarano que se anunciaron en prensa o en preparación ${ }^{11}$}

El menudo mundo de los hombres serios. Ensayos humoristicos. Memorias de un espectro, Relato.

El drama del subconsciente de Pérez, Novela.

Revisión del proceso de Satanás, Ensayo.

Don Quijote de Francia, Novela.

\section{Relatos de Benigno Bejarano en la revista Lecturas}

(IX-1929): Un fantasma contemporáneo, il. Castany, n. ${ }^{0}$ 100, pp. 1223-1227.

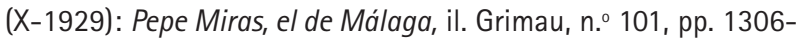
1311.

(II-1930): El milagro del séptimo, n. ${ }^{\circ} 105$, pp. 109 y 171-172.

(III-1930): Un golpe de fortuna, n. ${ }^{\circ} 106$, pp. 228-230 y 264.

(XI-1930): Los hijos del Milagro, il. Guasp, n. ${ }^{\circ} 114$, pp. 935-937.

(VI-1931): El misterio del anticuario, n. ${ }^{\circ} 121$, pp. 417-420 y 479.

(IV-1932): El caso del doctor González, il. Prat, n. ${ }^{131}$, pp. 345350.

\section{Textos sobre Benigno Bejarano}

Castañar, Fulgencio (julio 1976): "Un novelista olvidado. La sátira política de Benigno Bejarano", en Tiempo de Historia, 20, pp. 66-70.

Castañar, Fulgencio (septiembre-diciembre 1996): "Benigno Bejarano: periodismo de denuncia y literatura satírica. Perfil de un escritor extremeño olvidado", en Revista de Extremadura 21, pp. 151-163.

Labrador Ben, Julia Maria (2009): "Un héroe loco visita Marte. La novela festiva de Benigno Bejarano, un autor de literatura de combate", en Fidel López Criado (coord.): Héroes, mitos y monstruos en la literatura española contemporánea, Santiago de Compostela, Andavira, pp. 119-126.

Maya, Eduardo (2009): en Azagala, 10.

Obregón Roldán, Enrique de (14-VII-1994): "Sobre Benigno Bejarano", en "Cartas de los lectores", en La Vanguardia, p. 18.

Uribe, Augusto (2004): "El viaje a Marte de Bejarano (El secreto de un loco o El fin de una expedición sideral)", en Españoles que fueron a Marte, Madrid, edición del autor.

\section{Documentos}

(23-II-1981): Carta de J. Paniagua a Abel Marinel

\section{Artículos de prensa}

(20-IX-1924): "Indultos", en "La situación al día", en La Vanguardia, p. 6.

(15-VIII-1925): "Noticias militares", en La Vanguardia, p. 4.

(20-VII-1927): "Indultos", en "Notas del día", en La Vanguardia, p. 6.

\section{Estudios literarios}

Castañar, Fulgencio (1992): El compromiso en la novela de la II República, Madrid, Siglo XXI.

Coria, Javier (diciembre 2006): "Viajes literarios al espacio. Historia de las dicciones espaciales", en Barsoom, 1, pp. 5154.

Mañá Delgado, Gemma; García Heredero, Rafael; Monferrer Catalán, Luis; y Esteve Juárez, Luis A. (1997): La voz de los náufragos: La narrativa republicana entre 1936 y 1939, Madrid, Ediciones de la Torre. 
Labajo González, Trinidad (2003): Lecturas, Madrid, Consejo Superior de Investigaciones Científicas (Literatura Breve, 11).

\section{Estudios históricos}

Agramunt Lacruz, Francisco (junio 2003): "Intelectuales españoles en campos de exterminio", en Historia 16, 326, pp. 62-72.

Bermejo, Benito (2002): Francisco Boix, el fotógrafo de Mauthausen, Barcelona, RBA.

Bermejo, Benito y Checa, Sandra (2006): Libro memorial. Españoles deportados a los campos nazis (1940-1945), Madrid, Ministerio de Cultura.

López de Zuazo, Antonio (2007): Reseña de "Bermejo, Benito y Checa, Sandra, 2006: Libro memorial. Españoles deportados a los campos nazis (1940-1945), Madrid, Ministerio de Cultura, 588 páginas", en Estudios sobre el Mensaje Periodístico, 13, pp. 559-624.

Pons Prades, Eduardo (1995): Morir por la libertad. Españoles en los campos de exterminio nazis, Madrid, Vosa.

Pons Prades, Eduardo (2005): El holocausto de los republicanos españoles. Vida y muerte en los campos de exterminio alemanes (1940-1945), Barcelona, Belacqva (Colección El ojo de la Historia). Se trata de una reedición de Morir por la libertad.

Soriano, Ignacio y Madrid, Francisco (2008): Antología documental del anarquismo español, volumen $\mathrm{VI}$, Bibliografía e historiografía, tomo VI.I, Bibliografía del anarquismo en España. 1869-1939, Madrid.

\section{Libros de memorias}

Paz, Abel (1993): Entre la niebla (1939-1942), Barcelona, E[dición] A[utor], pp. 159-165.

\section{Libros de ficción}

Martínez Rizo, Alfonso (1932): 1945. El advenimiento del comunismo libertario. Una visión novelesca del porvenir, Valencia, Mañana.

\section{NOTAS}

1 Epifanio, diez años mayor que él, murió muy joven, cuando su mujer estaba embarazada de su tercer hijo. Dolores, la mediana, tuvo cinco hijos, uno de los cuales todavía vive, Segundo Moreno Bejarano.

2 Se le entregó la cartilla militar número 47873. Probablemente fue excluido en los reclutamientos anteriores por estrecho de pecho. En ese año falleció su padre.

Recibido: 2 de febrero de 2009

Aceptado: 23 de abril de 2009
3 Es el oficio que indica su Hoja de Reclutamiento, en la que también se dice que su religión es la católica.
4 Abel Paz la describe como "una madrileña muy echá palante" (Paz, 1993, 163) pero no nos indica su nombre. Por su sobrino-nieta Mertxe Antona Bejarano sabemos que se llamaba Conchita.

5 Abel Paz relata una anécdota muy curiosa sobre la rivalidad pictórica que surgió entre Eleuterio Blasco y Benigno Bejarano cuando éste, para ganar un poco de dinero, sin tener ni idea de pintura, comenzó a realizar copias para el marchante de Eleuterio. Al final no resultaron tales sino nuevos cuadros por las modificaciones que introdujo, pero al 
marchante le gustaron y se las pagó al doble de lo pactado, como originales. El problema surgió cuando el galerista encargó al propio Eleuterio sacar copias de ellos provocándole un enfado considerable (Paz, 1993, 64-65).

6 En casi todas las fuentes se ha indicado que Bejarano murió durante el invierno de 1944-1945, pero nos inclinamos a pensar que la fecha correcta es levemente anterior, verano de 1944, porque así lo indica alguien que con- vivió con él durante sus últimos meses, J. Paniagua, quien rememora en una carta fechada el 23 de febrero de 1981 lo duros que fueron sus últimos días en el campo de concentración. También Enrique de Obregón Roldan, con motivo del cincuentenario de su muerte, publicó el 14 de julio de 1994 en la sección de "Cartas de los lectores" del diario La Vanguardia un breve texto, "Sobre Benigno Bejarano", en el que afirmaba que la muerte había acaecido en verano.
7 Martínez Rizo, Alfonso (1932): 1945. El advenimiento del comunismo libertario. Una visión novelesca del porvenir, Valencia, Mañana.

8 Cuando tenía diecisiete años envió su primer artículo a un diario madrileño.

9 La edición de este libro fue financiada por el propio autor.

10 Véase nota 6.

11 Desconocemos si finalmente llegaron a publicarse. 